\title{
Modélisation 3D de l'os maxillaire dans l'analyse par éléments finis en implantologie orale : une nouvelle approche utilisant CBCT et anthropométrie
}

\author{
M. Hadj SaÏd (Marseille), L. Thollon (Marseille), Y. Godio-Raboutet (Marseille), J.H. \\ Catherine (Marseille), C.M. Chossegros (Marseille), D. Tardivo (Marseille)
}

Objectif : Caractériser l'os maxillaire postérieur chez l'adulte d'un point de vue géométrique pour obtenir des modèles numériques standards par éléments finis. Matériel et méthodes : Les images CBCT maxillaires des patients qui ont visité le service de Chirurgie Orale du CHU de La Timone à Marseille, France ont été recueillies au cours de l'année 2016. Les sujets inclus devaient être âgés de plus de 21 ans et être édentés au moins à partir de la première prémolaire maxillaire. Les patients atteints d'une pathologie osseuse ou d'un traitement influençant le remodelage osseux n'ont pas été inclus. La zone maxillaire postérieure a été définie pour chaque CBCT et 6 mesures de hauteur et de largeur de la crête alvéolaire ont été réalisées à l'aide d'une méthode anthropométrique. Une étude Gauge Anova $R \& R$ avec analyse de la répétabilité et de la reproductibilité de la variance des mesures, ainsi qu'une analyse en composantes principales (ACP) pour isoler des modèles standards, ont été menées. Les modèles 3D ont été réalisés à partir d'images au format DICOM. Résultats : Le CBCT de 100 hommes et 100 femmes ont été retenus dans notre étude. 1200 mesures de crête alvéolaire ont été réalisée et les valeurs moyennes de hauteur et de largeur des différentes parties de la zone maxillaire postérieure étaient très disparates. L'analyse statistique de variance a validé la répétabilité et la reproductibilité de notre protocole de mesures. L'ACP n'a pas permis d'identifier les modèles standards et ceux- ci ont été modélisés à partir de notre base de données. Conclusion : Notre travail est le premier à considérer des paramètres anthropométriques sur un large échantillon de sujets dans la méthode des éléments finis. Nous mettons ainsi en évidence la perspective de réaliser des modèles anatomiques complexes et réalistes à partir de l'anatomie humaine pour réaliser des tests biomécaniques en implantologie orale. 